\title{
Multidimensional Discrete Green's Function and Its Estimates
}

\author{
Yinsuo Jia* \\ School of Mathematics and Computer Science \\ Shangrao Normal University \\ Shangrao, China \\ Jiayinsuo2002@sohu.com \\ * Corresponding Author
}

\author{
Jinghong Liu \\ School of Mathematics and Computer Science \\ Shangrao Normal University \\ Shangrao, China \\ Ddliu1010@163.com
}

\begin{abstract}
This paper will discuss estimates for discrete Green's function of elliptic equations in dimensions seven and up. First, the definitions of some terms are given. Then the estimates for the regularized Green's function are derived. Finally, by using the triangular inequality, researchers obtain the estimates for discrete Green's function. The results of the paper play important roles in the research of superconvergence of finite element methods.
\end{abstract}

Keywords-Regularized Green's Function; Discrete Green's Function; Superconvergence; Finite Element; Ellptic Equations

\section{INTRODUCTION}

It is well known that estimates for the Green's function play very important roles in the study of the superconvergence (especially, pointwise superconvergence) of the finite element method (see [1-8]). For one- and two-dimensional elliptic problems, one has obtained many optimal estimates for the Green's function (see [1]). Recently, to solve the three-dimensional elliptic problems, the $W^{2,1}$-seminorm optimal estimate with order $O\left(|\ln h|^{\frac{2}{3}}\right)$ for the discrete Green's function and the $W^{1,1}$-seminorm optimal estimate with order $O\left(|\ln h|^{\frac{4}{3}}\right)$ for the discrete derivative Green's function were derived (see [5, 9]). It might seem that in current practical applications of the finite element method there is no need for simplicial higher-dimensional elements. Nevertheless, it is well known that for example in areas like financial mathematics, particle physics, statistical physics and general relativity, higher-dimensional pdes need to be solved (see [10]).

Therefore, it is meaningful to study the estimates for the Green's function in four and up space dimensions. Recently, for dimensions four to six, researchers have also considered the estimates for discrete Green's functions. In this paper, researchers will derive some estimates for the discrete Green's function for dimensions seven and up. Researchers will find that there are not the optimal estimates for discrete Green's functions other than those in dimensions one to six.
In this paper, researchers shall use the symbol $\mathrm{C}$ to denote a generic constant, which is independent from the discretization parameter $h$ and which may not be the same in each occurrence and also use the standard notations for the Sobolev spaces and their norms.

\section{BOUNDARY VALUE PROBLEM AND FINITE ELEMENTS DISCRETIZATION} is

The model problem that researchers study in this paper

$$
-\Delta u=f \text { in } \Omega, \quad u=0 \text { on } \partial \Omega \text {. }
$$

Where $\Omega \subset R^{d}(d \geq 7)$ is a bounded polytopic domain. Let $\left\{T^{h}\right\}$ be a regular family of uniform partitions of $\bar{\Omega}$, and $S_{0}^{h}(\Omega) \subset H_{0}^{1}(\Omega)$ the finite element space.

Discretizing the above elliptic equation using $S_{0}^{h}(\Omega)$ as approximating space means finding $u_{h} \in S_{0}^{h}(\Omega)$ such that $a\left(u_{h}, v\right)=(f, v)$ for all $v \in S_{0}^{h}(\Omega)$, where

$$
\begin{aligned}
& a\left(u_{h}, v\right) \equiv \int_{\Omega} \nabla u_{h} \cdot \nabla v d X \\
& (f, v) \equiv \int_{\Omega} f v d X .
\end{aligned}
$$

In addition, researchers also assume that the given function $\mathrm{f}$ is smooth enough. For every $Z \in \Omega$, researchers define the discrete $\delta$ function $\delta_{Z}^{h} \in S_{0}^{h}(\Omega)$, and the $L^{2}$-projection $P_{h} u \in S_{0}^{h}(\Omega)$ such that

$$
\begin{aligned}
& \left(v, \delta_{Z}^{h}\right)=v(Z) \forall v \in S_{0}^{h}(\Omega), \\
& \left(u-P_{h} u, v\right)=0 \quad \forall v \in S_{0}^{h}(\Omega) .
\end{aligned}
$$$$
\text { Let } G_{Z}^{*} \in H^{2}(\Omega) \subset H_{0}^{1}(\Omega) \text { be the solution of the }
$$
elliptic problem $-\Delta G_{Z}^{*}=\delta_{Z}^{h}$, Researchers may call $G_{Z}^{*}$ the regularized Green's function. Further, let the discrete 
Green's function $G_{Z}^{h} \in S_{0}^{h}(\Omega)$ be the finite element approximation to $G_{Z}^{*}$. Thus,

$$
a\left(G_{Z}^{*}-G_{Z}^{h}, v\right)=0 \quad \forall v \in S_{0}^{h}(\Omega) .
$$

The purpose of this paper is to bind the term $\left|G_{Z}^{h}\right|_{2,1, \Omega}^{h}$.

The rest of this paper is organized as follows. In Section 3 researchers derive the $W^{2,1}$-seminorm estimate for $G_{Z}^{*}$. Section 4 is devoted to the estimate for $G_{Z}^{h}$.

III. ESTIMATES FOR THE REGULARIZED GREEN'S FUNCTION $G_{Z}^{*}$

We first introduce the weight function defined by

$$
\phi \equiv \phi(X)=\left(|X-\bar{X}|^{2}+\theta^{2}\right)^{-\frac{d}{2}} \forall X \in \bar{\Omega},
$$

Where $\bar{X} \in \bar{\Omega}$ is a fixed point, $\theta=h h$, and $\gamma \in(d,+\infty)$ is a suitable real number.

For every $\alpha \in R$, researchers give the following notations:

$$
\begin{aligned}
& \left|\nabla^{n} v\right|^{2}=\sum_{|\beta|=n}\left|D^{\beta} v\right|^{2},\left|\nabla^{n} v\right|_{\phi^{\alpha}, \Omega}=\left(\int_{\Omega} \phi^{\alpha}\left|\nabla^{n} v\right|^{2} d X\right)^{\frac{1}{2}}, \\
& \|v\|_{m, \phi^{\alpha}, \Omega}^{2}=\sum_{n=0}^{m}\left|\nabla^{n} v\right|_{\phi^{\alpha}, \Omega}^{2},
\end{aligned}
$$

Where $\beta=\left(\beta_{1}, \cdots, \beta_{d}\right),|\beta|=\beta_{1}+\cdots+\beta_{d}$, and $\beta_{i} \geq 0, i=1, \cdots, d$ are integers. In particular, for the case of $m=0$, researchers write

$$
\|v\|_{\phi^{\alpha}, \Omega}=\left(\int_{\Omega} \phi^{\alpha}|v|^{2} d X\right)^{\frac{1}{2}} .
$$
that

We assume that there exists a $q_{0}\left(1<q_{0} \leq+\infty\right)$ such

$$
-\Delta: W^{2, q}(\Omega) \cap W_{0}^{1, q}(\Omega) \longrightarrow L^{q}(\Omega)\left(1<q<q_{0}\right)
$$

That is (the so-called a priori estimate)

$$
\|v\|_{2, q, \Omega} \leq C(q)\|-\Delta v\|_{0, q, \Omega},
$$

Where $C(q)$ denotes a constant depending on $q$. The weight function $\phi$ possesses the following properties:

$$
\begin{gathered}
\left|\nabla^{n} \phi^{\alpha}\right| \leq C(\alpha, n) \phi^{\alpha+\frac{n}{d}}, \alpha \in \mathcal{R}, n=1,2 . \\
\int_{\Omega} \phi^{\alpha} d X \leq C(\alpha-1)^{-1} \theta^{-d(\alpha-1)} \forall \alpha>1, \\
\int_{\Omega} \phi d X \leq C(k)|\ln \theta|, \theta \leq k<1 .
\end{gathered}
$$

In addition, researchers have for the $L^{2}$-projection operator

$$
\left\|P_{h} w\right\|_{1, q, \Omega} \leq C(q)\|w\|_{1, q, \Omega} \text { for } 1 \leq q \leq \infty .
$$

In the following, researchers will give some estimates for the regularized Green's function.

Lemma 1. For $\delta_{Z}^{h}$ the discrete $\delta$ function defined by (4) (2), researchers have the weighted-norm estimate

$$
\left\|\delta_{Z}^{h}\right\|_{\phi^{-\alpha}} \leq C h^{\frac{d(\alpha-1)}{2}} \forall \alpha>0 .
$$

Proof. Obviously,

$$
\begin{aligned}
\left\|\delta_{Z}^{h}\right\|_{\phi^{-\alpha}}^{2} & \leq C \int_{\Omega}\left(|X-Z|^{2}+\theta^{2}\right)^{\frac{d \alpha}{2}} h^{-2 d} e^{-C h^{-1}|X-Z|} d X \\
& \leq C \int_{0}^{\infty}\left(r^{2}+\theta^{2}\right)^{\frac{d \alpha}{2}} h^{-2 d} e^{-C h^{-1} r} r^{d-1} d r .
\end{aligned}
$$

Set $h^{-1} r=t$, thus

$$
\begin{aligned}
\left\|\delta_{Z}^{h}\right\|_{\phi^{-\alpha}}^{2} & \leq C h^{(\alpha-1) d} \int_{0}^{\infty}\left(t^{2}+\gamma^{2}\right)^{\frac{d \alpha}{2}} e^{-C t} t^{d-1} d t \\
& \leq C h^{(\alpha-1) d} .
\end{aligned}
$$

Further,

$$
\left\|\delta_{Z}^{h}\right\|_{\phi^{-\alpha}}^{2} \leq C h^{d(\alpha-1)}
$$

Which is the result (10).

Lemma 2. For $q_{0}>2$ and $v \in H^{2}(\Omega) \cap H_{0}^{1}(\Omega)$, There exists a constant $C=C(\alpha, \Omega)>0$ such that $\left\|\nabla^{2} v\right\|_{\phi^{-\alpha}}^{2} \leq C\left(\|\Delta v\|_{\phi^{-\alpha}}^{2}+|v|_{1, \phi^{-\alpha+\frac{2}{d}}}^{2}+\|v\|_{\phi^{-\alpha+\frac{4}{d}}}^{2}\right) \forall \alpha \in \mathcal{R}$.

Proof.

$$
\begin{aligned}
& \left\|\nabla^{2} v\right\|_{\phi^{-\alpha}}^{2}=\int_{\Omega} \phi^{-\alpha}\left|\nabla^{2} v\right|^{2} d x=\int_{\Omega}\left(\phi^{-\frac{\alpha}{2}}\left|\nabla^{2} v\right|\right)^{2} d x \\
\leq & C\left(\int_{\Omega}\left|\nabla^{2}\left(\phi^{-\frac{\alpha}{2}} v\right)\right|^{2} d x+\int_{\Omega}\left|v \nabla^{2} \phi^{-\frac{\alpha}{2}}\right|^{2} d x+\int_{\Omega}\left|\nabla \phi^{-\frac{\alpha}{2}}\right|^{2}|\nabla v|^{2} d x\right) \\
\leq & C\left(\left\|\nabla^{2}\left(\phi^{-\frac{\alpha}{2}} v\right)\right\|_{0}^{2}+\|v\|_{\phi^{-\alpha+\frac{4}{a}}}^{2}+|v|_{1, \phi^{-\alpha+\frac{2}{\alpha}}}^{2}\right) \\
\leq & C\left(\left\|\Delta\left(\phi^{-\frac{\alpha}{2}} v\right)\right\|_{0}^{2}+\|v\|_{\phi^{-\alpha+\frac{4}{a}}}^{2}+|v|_{1, \phi^{-\alpha+\frac{2}{a}}}^{2}\right) \\
\leq & C\left(\int_{\Omega} \phi^{-\alpha}|\Delta v|^{2} d x+\int_{\Omega}\left|\nabla \phi^{-\frac{\alpha}{2}}\right|^{2}|\nabla v|^{2} d x\right. \\
& \left.+\int_{\Omega}\left|\Delta \phi^{-\frac{\alpha}{2}}\right|^{2}|v|^{2} d x+\|v\|_{\phi^{-\alpha+\frac{4}{d}}}^{2}+|v|_{1, \phi^{-\alpha+\frac{2}{d}}}^{2}\right) \\
\leq & C\left(\|\Delta v\|_{\phi^{-\alpha}}^{2}+|v|_{1, \phi^{-\alpha+\frac{2}{d}}}^{2}+\|v\|_{\phi^{-\alpha+\frac{4}{d}}}^{2}\right) .
\end{aligned}
$$

Lemma 3. Suppose $q_{0}>2$ and $\alpha \in R$. For $G_{Z}^{*}$ and $\delta_{Z}^{h}$, the regularized Green's function and the discrete $\delta$ function, respectively, researchers have

$$
\left\|\nabla^{2} G_{Z}^{*}\right\|_{\phi^{-\alpha}}^{2} \leq C\left\|\delta_{Z}^{h}\right\|_{\phi^{-\alpha}}^{2}+C\left\|G_{Z}^{*}\right\|_{\phi^{-\alpha+\frac{4}{d}}}^{2} .
$$

$G_{Z}^{*}$, researchers have

$$
\begin{aligned}
\left\|\nabla^{2} G_{z}^{*}\right\|_{\phi^{-\alpha}}^{2} & \leq C\left(\left\|\Delta G_{z}^{*}\right\|_{\phi^{-\alpha}}^{2}+\left|G_{z}^{*}\right|_{1, \phi^{-\alpha+\frac{2}{d}}}^{2}+\left\|G_{z}^{*}\right\|_{\phi^{-\alpha+\frac{4}{d}}}^{2}\right) \\
& \leq C\left\|\delta_{z}^{h}\right\|_{\phi^{-\alpha}}^{2}+C\left|a\left(G_{z}^{*}, \phi^{-\alpha+\frac{2}{d}} G_{z}^{*}\right)\right|+C\left\|G_{z}^{*}\right\|_{\phi^{-\alpha+\frac{4}{a}}}^{2} \\
& \leq C\left\|\delta_{z}^{h}\right\|_{\phi^{-\alpha}}^{2}+C\left|\left(\delta_{z}^{h}, \phi^{-\alpha+\frac{2}{a}} G_{z}^{*}\right)\right|+C\left\|G_{z}^{*}\right\|_{\phi^{-\alpha+\frac{4}{d}}}^{2} \\
& \leq C\left\|\delta_{z}^{h}\right\|_{\phi^{-\alpha}}^{2}+C\left\|\delta_{z}^{h}\right\|_{\phi^{-\alpha}}\left\|G_{z}^{*}\right\|_{\phi^{-\alpha+\frac{4}{d}}}+C\left\|G_{z}^{*}\right\|_{\phi^{-\alpha+\frac{4}{d}}}^{2} \\
& \leq C\left\|\delta_{z}^{h}\right\|_{\phi^{-\alpha}}^{2}+C\left\|G_{z}^{*}\right\|_{\phi^{-\alpha+\frac{4}{d}}}^{2},
\end{aligned}
$$


Which completes the proof of the result (12).

Lemma 4. Suppose $q_{0}>2$ and $\alpha$ satisfying $\frac{4}{d}-1<\alpha<\frac{4}{d}-1+\min \left\{1, \frac{d}{2}-1\right\}$. Then for $G_{Z}^{*}$ and $\delta_{Z}^{h}$, the regularized Green's function and the discrete $\delta$ function, respectively, researchers have

$$
\left\|\nabla^{2} G_{Z}^{*}\right\|_{\phi^{-\alpha}}^{2} \leq C h^{d(\alpha-1)} .
$$

Theorem 1. Suppose $q_{0}>2$ and $d \geq 7$. For $G_{Z}^{*}$, the regularized Green's function, researchers have

$$
\left\|G_{Z}^{*}\right\|_{2,1} \leq C h^{2-\frac{d}{2}} .
$$

Proof. Obviously,

$$
\left\|G_{Z}^{*}\right\|_{2,1}^{2} \leq \int_{\Omega} \phi^{\alpha} d X \cdot\left\|\nabla^{2} G_{Z}^{*}\right\|_{\phi^{-\alpha}}^{2} .
$$

It is easy to prove

$$
\int_{\Omega} \phi^{\alpha} d X \leq C(1-\alpha)^{-1} \forall 0<\alpha<1
$$
(13),

Thus when $\frac{4}{d}-1<\alpha<\frac{4}{d}-1+\min \left\{1, \frac{d}{2}-1\right\}$, from

$$
\left\|G_{Z}^{*}\right\|_{2,1}^{2} \leq C \frac{h^{d(\alpha-1)}}{1-\alpha} .
$$

Hence researchers have

$$
\left\|G_{Z}^{*}\right\|_{2,1}^{2} \leq C \inf _{\frac{4}{d}-1<\alpha<\frac{4}{d}} \frac{h^{d(\alpha-1)}}{1-\alpha}=C h^{4-d} .
$$

The result (14) is obtained.

\section{ESTIMATES FOR THE DISCRETE GREEN'S FUNCTION}

$$
G_{Z}^{h}
$$

Lemma 4. Suppose $q_{0}>2$, researchers have

$$
\left\|G_{Z}^{*}-G_{Z}^{h}\right\|_{1, \phi^{-\alpha}}^{2} \leq C h^{2+d(\alpha-1)} .
$$

Where

Proof.

$$
\max \left\{\frac{4}{d}-1,0\right\}<\alpha<\min \left\{\frac{4}{d}, \frac{4}{d}+\frac{d}{2}-2,1-\frac{2}{q_{0}}+\frac{2}{d}\right\} .
$$

$$
\begin{aligned}
& \left\|G_{Z}^{*}-G_{Z}^{h}\right\|_{1, \phi^{-\alpha}}^{2} \\
\leq & C h^{2}\left\|\nabla^{2}\left(G_{Z}^{*}\right)\right\|_{\phi^{-\alpha}}^{2}+C\left\|G_{Z}^{*}-G_{Z}^{h}\right\|_{\phi^{-\alpha+\frac{2}{d}}}^{2} \\
\leq & \hat{C}\left(h^{2}\left\|\nabla^{2}\left(G_{Z}^{*}\right)\right\|_{\phi^{-\alpha}}^{2}+\left\|G_{Z}^{*}-G_{Z}^{h}\right\|_{\phi^{-\alpha+\frac{2}{d}}}^{2}\right) .
\end{aligned}
$$

In addition, researchers get

$$
\left\|G_{Z}^{*}-G_{Z}^{h}\right\|_{\phi^{-\alpha+\frac{2}{d}}}^{2} \leq \frac{2}{3 \hat{C}}\left\|G_{Z}^{*}-G_{Z}^{h}\right\|_{1, \phi^{-\alpha}}^{2} .
$$

Then researchers have

\section{ACKNOWLEDGMENTS}

This work was supported by the National Natural Science Foundation of China Grant 11161039.

$$
\left\|G_{Z}^{*}-G_{Z}^{h}\right\|_{1, \phi^{-\alpha}}^{2} \leq C h^{2}\left\|\nabla^{2}\left(G_{Z}^{*}\right)\right\|_{\phi^{-\alpha}}^{2} .
$$

From (13)

$$
\left\|\nabla^{2}\left(G_{Z}^{*}\right)\right\|_{\phi^{-\alpha}} \leq C h^{\frac{d(\alpha-1)}{2}} .
$$

By the above inequalities, researchers immediately obtain the result (15).

Lemma 5. Suppose $d \geq 7$ and $q_{0} \geq \frac{2 d}{d-2}$. For $G_{Z}^{*}$ and $G_{Z}^{h}$, the regularized Green's function and the discrete Green's function, respectively, researchers have

$$
\left\|G_{Z}^{*}-G_{Z}^{h}\right\|_{1,1} \leq C h^{3-\frac{d}{2}} .
$$

Proof. Obviously,

$$
\left\|G_{Z}^{*}-G_{Z}^{h}\right\|_{1,1}^{2} \leq \int_{\Omega} \phi^{\alpha} d X \cdot\left\|G_{Z}^{*}-G_{Z}^{h}\right\|_{1, \phi^{-\alpha}}^{2} .
$$

Since

$$
\int_{\Omega} \phi^{\alpha} d X \leq C(1-\alpha)^{-1} \forall 0<\alpha<1
$$

When $d \geq 7$ and $q_{0} \geq \frac{2 d}{d-2}$, from (16) researchers have $0<\alpha<\frac{4}{d}<1$. Thus from (15) researchers get

$$
\left\|G_{Z}^{*}-G_{Z}^{h}\right\|_{1,1}^{2} \leq C \inf _{\alpha} \frac{h^{2+d(\alpha-1)}}{1-\alpha}=C h^{6-d} .
$$

Namely

$$
\left\|G_{Z}^{*}-G_{Z}^{h}\right\|_{1,1} \leq C h^{3-\frac{d}{2}} .
$$

The result (17) is obtained.

Theorem 2. Suppose $d \geq 7$ and $q_{0} \geq \frac{2 d}{d-2}$, researchers have

$$
\left\|G_{Z}^{h}\right\|_{2,1}^{h} \leq C h^{2-\frac{d}{2}} .
$$

Proof. Obviously,

$$
\begin{aligned}
\left\|G_{Z}^{*}-G_{Z}^{h}\right\|_{2,1}^{h} & \leq\left\|G_{Z}^{*}-\Pi G_{Z}^{*}\right\|_{2,1}^{h}+\left\|\Pi G_{Z}^{*}-G_{Z}^{h}\right\|_{2,1}^{h} \\
& \leq C\left\|G_{Z}^{*}\right\|_{2,1}+C h^{-1}\left\|\Pi G_{Z}^{*}-G_{Z}^{h}\right\|_{1,1} \\
& \leq C\left\|G_{Z}^{*}\right\|_{2,1}+C h^{-1}\left\|\Pi G_{Z}^{*}-G_{Z}^{*}\right\|_{1,1}+C h^{-1}\left\|G_{Z}^{*}-G_{Z}^{h}\right\|_{1,1} \\
& \leq C\left\|G_{Z}^{*}\right\|_{2,1}+C h^{-1}\left\|G_{Z}^{*}-G_{Z}^{h}\right\|_{1,1},
\end{aligned}
$$

Where $\Pi$ is an interpolation operator. By the triangular

Inequality,

$\left\|G_{Z}^{h}\right\|_{2,1}^{h} \leq\left\|G_{Z}^{*}-G_{Z}^{h}\right\|_{2,1}^{h}+\left\|G_{Z}^{*}\right\|_{2,1} \leq C\left\|G_{Z}^{*}\right\|_{2,1}+C h^{-1}\left\|G_{Z}^{*}-G_{Z}^{h}\right\|_{1,1}$,

From (14), (17) and (19), researchers immediately obtain the result (18). 


\section{REFERENCES}

[1] Q. D. Zhu and Q. Lin, "Superconvergence theory of the finite element methods (in Chinese)," Changsha: Hunan Science and Technology Press, 1989.

[3] C. M. Chen, "Construction theory of superconvergence of finite elements (in Chinese)," Changsha: Hunan Science and Technology Press, 2001.

[4] G. Goodsell, "Pointwise superconvergence of the gradient for the linear tetrahedral element," Numer. Meth. Part. Differ. Equ., Vol. 10,1994 , pp. 651-666.

[5] J. H. Liu, B. Jia, and Q. D. Zhu, "An estimate for the threedimensional discrete Green's function and applications," J. Math. Anal. Appl., Vol. 370, 2010, pp. 350-363.

[6] J. H. Liu and Q. D. Zhu, "Uniform superapproximation of the derivative of tetrahedral quadratic finite element approximation,” J. Comput. Math., Vol. 23, 2005, pp. 75-82.

[7] J. H. Liu and Q. D. Zhu, "Maximum-norm superapproximation of the gradient for the trilinear block finite element," Numer. Meth. Part. Differ. Equ., Vol. 23, 2007, pp. 1501-1508.
[2] C. M. Chen and Y. Q. Huang, "High accuracy theory of finite element methods (in Chinese)," Changsha: Hunan Science and Technology Press, 1995.

[8] J. H. Liu and Q. D. Zhu, "Pointwise supercloseness of tensorproduct block finite elements," Numer. Meth. Part. Differ. Equ., Vol. 25 , 2009, pp. 990-1008.

[9] J. H. Liu and Q. D. Zhu, "The estimate for the W1,1-seminorm of discrete derivative Green's function in three dimensions (in Chinese)," J. Hunan Univ. Arts Sci., Vol. 16, 2004, pp. 1-3.

[10] J. H. Brandts and M. Krizek, "Gradient superconvergence on uniform simplicial partitions of polytopes," IMA. J. Numer. Anal., Vol. 23, 2003, pp. 489-505.

[11] J. H. Liu and Y. S. Jia, "Five-Dimensional Discrete Green's Function and Its Estimates," J. Comp. Anal. Appl., Vol. 18 2015, pp. 620-627. 